\title{
Atypical Peripheral Bronchial Carcinoid
}

\author{
S SHARMA, R MAHAJAN, A NEGI, D GOYAL, N PRASHER, A JHOBTA
}

Ind J Radiol Imag 2006 16:2:211-214

\section{Key words : Carcinoid, bronchus, CT, Chest radiograph}

\section{Introduction :}

Bronchial carcinoids are uncommon, low grade, slow growing, malignant neoplasms comprising $5 \%$ of all primary lung cancers and originally called as bronchial adenomas. Bronchial carcinoids are thought to arise from neuroendocrine / Kulchitsky's cells located in bronchial mucosa. They range from low-grade typical carcinoids to more aggressive atypical carcinoids. Bronchial carcinoids therefore demonstrate a wide spectrum of clinical behavior and histological features. Peripheral bronchial carcinoids appear as solitary nodules. Central bronchial carcinoids manifests as an endobronchial nodule or hilar/ perihilar mass with a close anatomic relationship to the bronchus. On contrary to typical carcinoids, atypical carcinoids have different histologocal features that suggest aggressive nature with worse prognosis.

This report documents the case of atypical peripheral bronchial carcinoid in a young female in perpeurium.

\section{Case Report:}

A twenty year old woman in her perpeurium presented with dyspnoea, left sided chest pain of one month duration. General and systemic examinations were normal. Erythrocyte sedimentation rate was $95 \mathrm{~mm}$ in first hour. Beta human chorionic gonadotrophin was normal.

On chest radiograph, mediastinum was pushed to the right. A huge homogeneous opacity was seen occupying the middle and lower zones of left hemi thorax. The superior margin of opacity was well defined and was making an acute angle with the chest wall. Medial border of the opacity was making a silhouette with left heart border. No calcification was seen in the mass. The left costo-phrenic angle was obliterated. Rib cage was normal (Fig1).

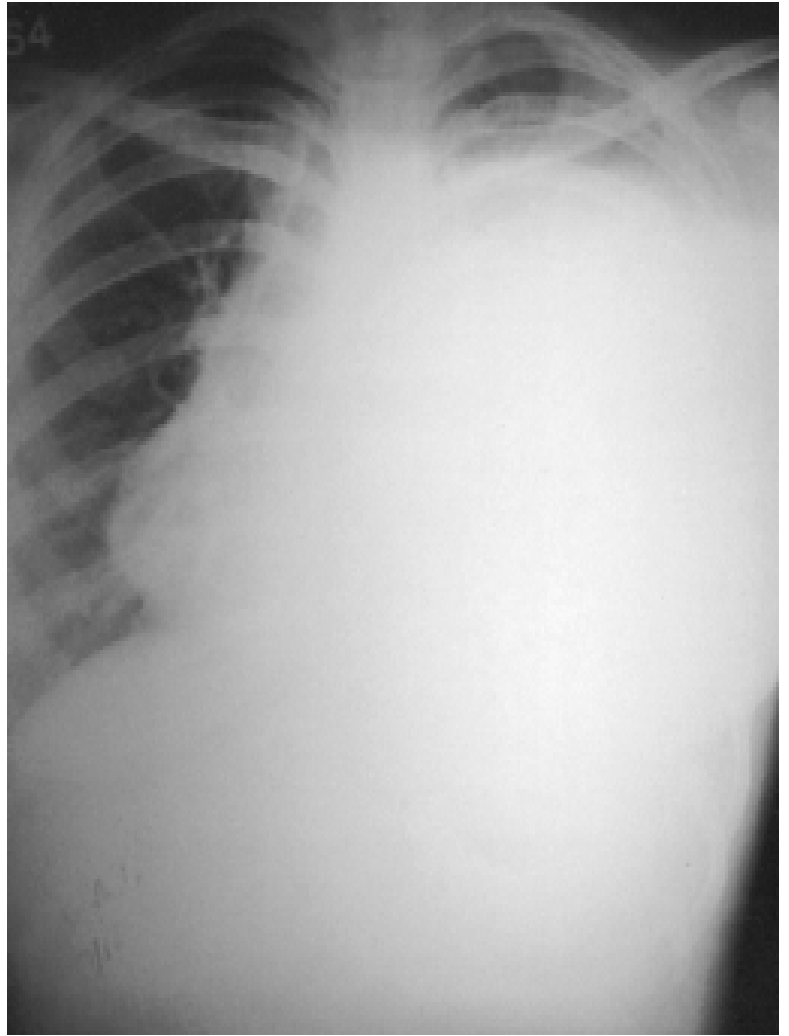

Fig. 1: Chest radiograph PA view showing a huge homogeneous opacity occupying the middle and lower zones of left hemi thorax.

Computed tomography of thorax revealed a well defined, huge heterogeneous mass occupying the left hemi thorax. Mass involved anterior segment of left upper lobe, lingular and left lower lobe. Mass was making acute angle with the chest wall and was running parallel to the left main bronchus. The bronchus per se was normal though pushed postero-medially. The density of mass ranged from $24 \mathrm{HU}$ to $48 \mathrm{HU}$ (Fig 2). After I.V contrast, the mass showed heterogeneous enhancement with some well-defined focal nodules and linear bands of marked enhancement. There was no mediastinal lymphadenopathy or pleural effusion (Fig 3).

From the Radiodiagnosis Deptt. Pathology Deptt. Indira Gandhi Medical College, Shimla - 171001 (H.P.)

Request for Reprints: Dr. Sanjiv Sharma, 1007, Basant Vihar, Kasumpti, Shimla. (H.P.) - 171009

Received 15 October 2005; Accepted 20 February 2006 
212 S Sharma et al

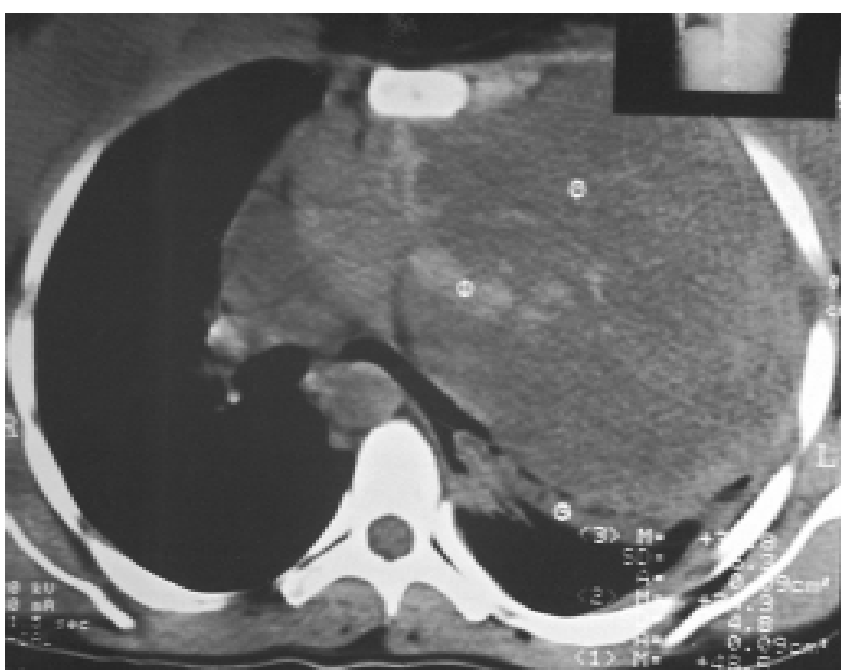

Fig. 2: Plain computed tomography of thorax showing a well defined, huge heterogeneous mass occupying the left hemi thorax.

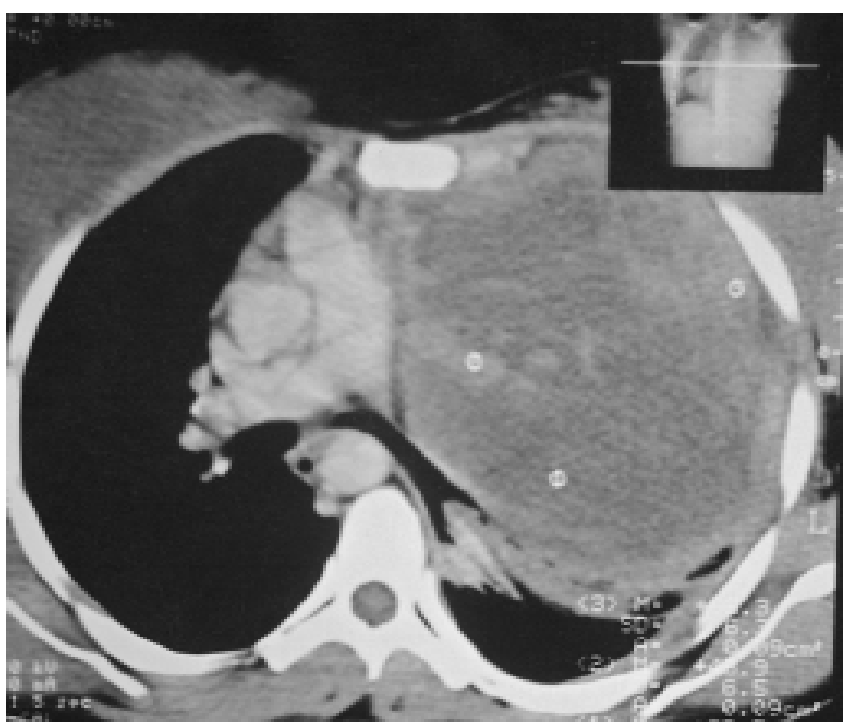

Fig.3: Contrast computed tomography of thorax showing the mass with heterogeneous enhancement, some well-defined focal nodules and linear bands of marked enhancement.

Fine needle aspiration cytology from the mass revealed capillaries surrounded by spindle cells with blunt ended nuclei. The cells had moderate amount of cytoplasm. Chromatin of cells had salt and pepper like appearance. Findings were confirmatory of atypical peripheral bronchial carcinoid.

Patient refused surgery and therefore was put on combination chemotherapy with high dose radiotherapy. She underwent two cycles of combination chemotherapy with radiotherapy. Follow up chest radiograph after six months revealed reduction in the size of the mass with reduced density in the upper half of the mass. Mediastinum reverted to its normal position (Fig 4). Follow up computed tomography of thorax also showed reduced size of mass. There was considerable increase in the low attenuation / necrotic component of the mass with density of around
$12 \mathrm{HU}$. The predominant low attenuation component of the mass showed no enhancement. The thick intervening septae traversing the mass showed significant enhancement. Solid enhancing nodules seen in the pre treatment scans were also no longer visible. No mediastinal lymphadenopathy or pleural effusion was seen. The left main bronchus was not infiltrated by the mass (Fig 5).

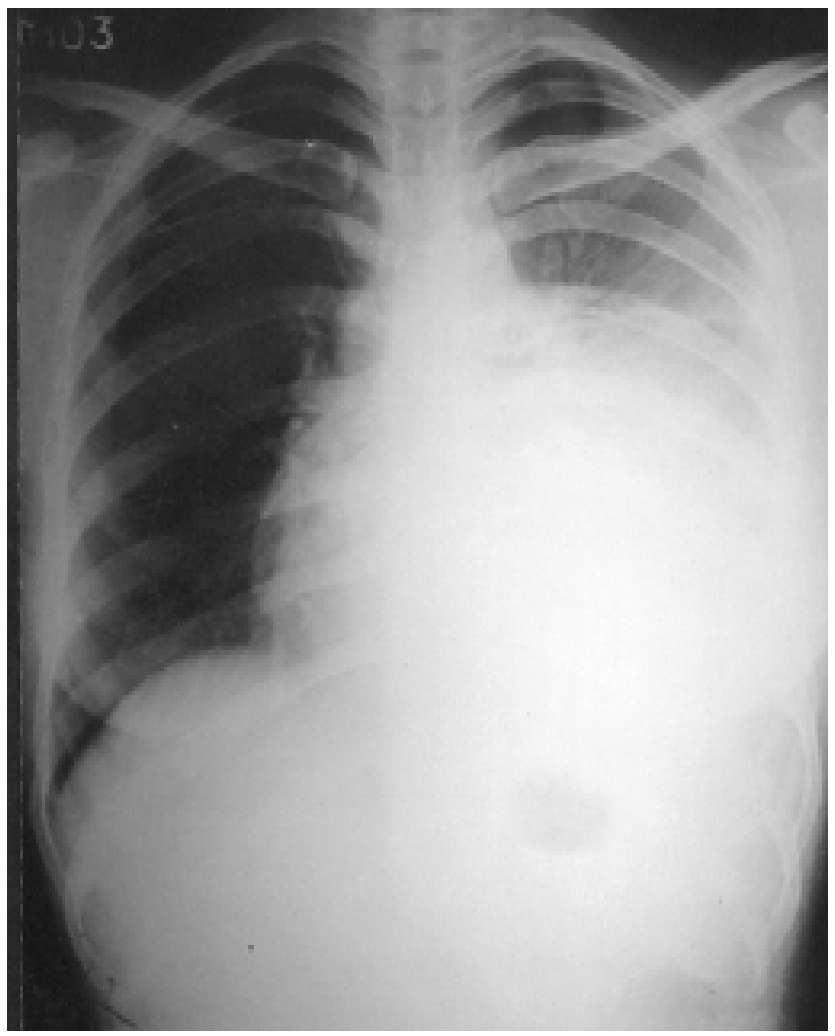

Fig. 4: Chest radiograph $\mathrm{PA}$ view showing reduction in the size of the mass with reduced density in the upper half of the mass. Mediastinum is seen at its normal position

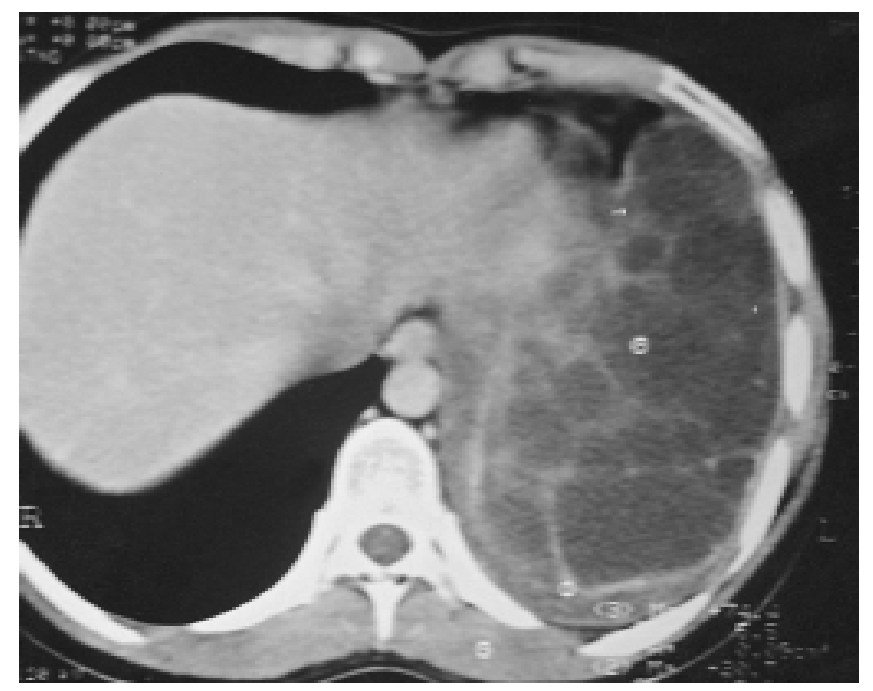

Fig. 5: Contrast computed tomography of thorax showing reduction in the size of mass. Increase in the necrotic component of the mass is also seen. 
Ultrasound of thorax and abdomen was done twenty days after the follow up computed tomography. Left hemi-thorax revealed a multiseptated hypoechoic / fluid density mass with spoke wheel appearance. Minimal pleural effusion was also detected on left side. Ultrasound of abdomen was unremarkable and showed no evidence of metastases (Fig 6).

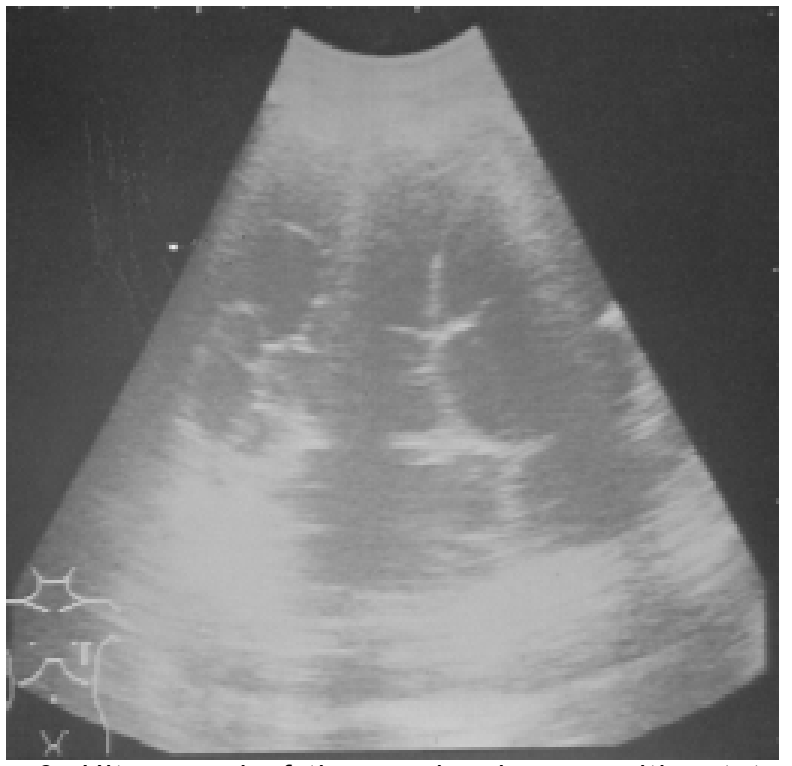

Fig. 6: Ultrasound of thorax showing a multiseptated hypoechoic / fluid density mass with spoke wheel appearance. Minimal pleural effusion is also seen on left side.

Patient is being followed up for last ten months. She had shown marked deterioration in physical well being without any evidence of metastases on systemic and radiological examination.

\section{Discussion :}

Bronchial carcinoids are neoplasms of bronchial endocrine or APUD cells derived from primitive gut. They account for $5 \%$ of all primary lung cancers. They are slow growing tumors which affect two persons per million per year. The age range is wide from teenagers to old age. There is no relationship to smoking, environmental, inhalation of carcinogens or radiation. There is slight female predominance $[1,7]$.

Bronchial carcinoids usually arise from a main or segmental bronchus. Occasionaly the tumor arises in the peripheral lung. Histologically it is composed of aggregates of small cells with dark central nuclei and pale cytoplasm in a vascular stroma. So-called atypical carcinoid tumors show increased mitotic activity, irregular nuclei and prominent nucleoli with areas of tissue necrosis. This histology of atypical carcinoids is responsible for there aggressive behaviour. Occasionally amyloid material or bone may be laid down in stroma of carcinoid tumor [2].
According to site of origin, carcinoids are divided into fore gut, mid gut and hind gut tumors. According to histology they are classified as typical or atypical carcinoids. Typical carcinoids are more common (90\%), perihilar in location, show indolent course, have less tendency to metastasize and calcify in $25 \%$ of cases. Five-year survival rate is more than $90 \%$. Atypical carcinoids are peripheral in location, larger than typical carcinoids, aggressive in nature with tendency to metastasize in $30 \%$ to $50 \%$ of cases. Calcification is uncommon in atypical peripherally located carcinoids [3].

Peripheral carcinoids are usually asymptomatic. The centrally located carcinoids cause partial / complete bronchial obstruction. This leads to symptoms such as cough , wheeze, infections, atelaecetasis. Cushing's and carcinoid syndromes are rare $(1 \%)$ with bronchial carcinoids [2, 4].

Chest radiograph is abnormal in $75 \%$ to $90 \%$ of cases. Peripheral carcinoids may present as simple peripheral pulmonary nodule. Central hilar / perihilar mass with or without atelectasis, bronchieatasis, mucoid impaction or consolidation can be seen. The majorities of bronchial carcinoids arise in the large bronchi and cause partial or complete obstruction.

Computed tomography discloses small tumors that are occult on plain radiographs. The appearance differs from carcinoma in that the bronchus may widen slightly as it approaches the mass. The most frequent presentation is hilar / perihilar, well circumscribed, rounded or ovoid mass which may have a notched or lobulated contour. It ranges in size from 2 to $5 \mathrm{~cm}$. Non-spherical lesions are oriented parallel to the axis of adjacent major bronchi or pulmonary artery. Calcification is uncommon in peripheral bronchial carcinoids as compared to central variety. Because carcinoids have a rich vascular stroma , they show homogeneous enhancement. Lymphadenopathy and distant metastasis are also less common with peripheral bronchial carcinoids $[3,5,6]$.

Carcinoid tumors should be removed surgically. Tumors confined to bronchus are treated by sleeve resection. Other tumors may require lobectomy or pneumonectomy.After surgery the prognosis is very good with ten years survival rate upto $90 \%$. In present case patient refused surgery resulting in poorer outcome. Liver metastases if present are treated by arterial embolisation. Symptoms of carcinoid syndrome are treated with serotonin antagonists. Radiotherapy and chemotherapy can be used as adjuvant to destroy tumor cells and reduce the tumor bulk [ 8 ].

\section{References:}

1. Moertel CG, Karnofsky memorial lecture, An odyssey in 
the land of small tumors. J Clin Oncol. 1994; 5:15032.

2. Davila DG, Dunn WF, Tazelaar HD, et al , Bronchial carcinoid tumors. Mayo Clin Proc. 1993; 68:795-803.

3. Arrigoni MG, Woolner LB, Bernatz PE , Atypical carcinoid tumors of the lung. J Thorac Cardiovasc Surg. 1972;64:413-421.

4. Fink G, Krelbaum T, Yellin A, et al, Pulmonary carcinoid: Presentation, diagnosis, and outcome in 142 cases in Israel and review of 640 cases from the literature. Chest. 2001; 119:1647-1651.

5. Thomas CF Jr, Tazelaar HD, Jett JR, Typical and atypical pulmonary carcinoids: Outcome in patients presenting with regional lymph node involvement. Chest. 2001;
119:1143-1150.

6. Davis SD, Zirn JR, Govoni AF, Peripheral carcinoid tumor of the lung: CT diagnosis. AJR Am J Roentgenol. 1990;155:1185-1187.

7. Davila DG, Dunn WF, Tazelaar HD, et al, Bronchial carcinoid tumors. Mayo Clin Proc. 1993;68:795-803.

8. Moertel, CG, Hanley, JA, Combination chemotherapy trials in metastatic carcinoid tumor and the malignant carcinoid syndrome. Cancer Clin Trials. 1979;2:327. 